\title{
Resonances of Microstructured Photonic Waveguides and Higher Order Homogenization Expansion
}

\author{
S.E. Golowich and M.I. Weinstein \\ Mathematical Sciences Research \\ Bell Laboratories, Lucent Technologies \\ Murray Hill, NJ 07974 \\ fax 9085823340
}

\begin{abstract}
We derive a homogenization expansion approach to obtaining the leaky modes, or scattering resonances, of photonic waveguides whose transverse structures are $N$ fold rotationally symmetric. Examples include microstructured, or "holey," optical fibers with air holes arranged in one or more concentric rings. We numerically calculate the leading order correction to the complex effective indexes for the leaky modes of various microstructured photonic waveguides in the scalar approximation. We observe that in many instances this two-term truncation of the homogenization expansion gives very good agreement with full simulations, even for fairly small values of $N$.
\end{abstract}

Key words: holey fiber, photonic band gap, photonic crystal, scattering frequency, scattering resonance, quasi-mode, radiation, leaky mode, tunneling loss

\section{Introduction}

There is currently a great deal of interest in the propagation of light in microstructured, or "holey," optical fiber waveguides with novel cross-sections consisting of holes surrounded by glass. The holes may be empty or filled with a material chosen to influence the propagation. The ability to vary the transverse geometry due to advances in fabrication technology, combined with the

Email address: miw@research.bell-labs.com (S.E. Golowich and M.I. Weinstein). 
large index contrasts possible with such structures, give multiple new degrees of freedom that potentially enable designs with radically different properties than is possible with standard fiber.

Numerous interesting phenomena have already been observed in such waveguides. Among them are (i) guiding by the interference based photonic band gap effect in fibers with an air core and (truncated) transverse periodic lattice of air holes [3]; (ii) variability of chromatic dispersion with microstructure [12], [7]; and resulting (iii) nonlinear effects in newly accessible spectral ranges due to microstructure-induced shifting zero dispersion point in glass core fiber $[12]$.

Efficient and accurate mathematical modeling of light propagation in microstructured fiber is clearly necessary for their design and analysis. A feature of most such structures is that they are inherently leaky due to the existence of paths leading from the core to the cladding that avoid the holes and pass only through the background glass. Such structures support no true guided modes, but they will have leaky modes characterized by complex-valued propagation constants or effective indexes (scattering resonances); the leakage rate is given by the imaginary part. Physically, this leakage is due to a combination of tunneling through the holes and propagating through the glass surrounding them. The ability to calculate such rates is clearly of fundamental importance. Other quantities of interest include the real parts of the effective indexes, which determine the response of the structure to longitudinal variations such as gratings, and the dispersion relations of the various leaky modes, which may be quite unusual compared to standard waveguides due to the presence of large index contrasts and interference effects.

There have been many numerical studies of microstructure fiber based on direct numerical calculation of static Maxwell's equations in order to determine the system's modes; some of them are also able to capture the attenuation rates. A variety of methods have been used, among them the multipole expansion [16], which works well for structures with circular holes; more general expansions in local bases [9] and Fourier decompositions [11], which are applicable to more general geometries; and scalar and vector beam propagation $[4,16]$, which are applicable to general geometries but have limitations in computing very small attenuation rates and have proven problematic in some geometries [15]. All of these techniques have the characteristic that the computational difficulty of the calculations increase with the complexity of the structures. By contrast, [1] show that for fine enough microstructure, an angularly averaged index profile gives accurate values for the real part of the effective indexes of the leaky modes; the leakage rates, however, are often greatly underestimated.

This paper summarizes recent work by the authors on the analysis and nu- 
merical computation of scattering resonances using ideas from multiple scale perturbation and homogenization theory. An expanded version of this work containing all details is in [6]. A rigorous mathematical theory is presented by the authors in [5]. We develop a multiple scale perturbation theory of a large class of "sufficiently oscillatory" structures suitable for the analytical study and efficient numerical computation of such quantities as leakage rates, group velocities, and dispersion, that becomes more accurate as the transverse structure becomes more oscillatory. The present study is carried out in the scalar approximation. If the transverse structure is invariant under rotation by $2 \pi / N$ (e.g. in the case of a single ring of $N$ holes uniformly distributed in an annulus) we expand the leaky modes and the effective indexes (scattering resonances) in powers of $1 / N$. The leading order in our expansion turns out to be the averaged or homogenized index profile $[2,8]$, referred to above. In this study, we focus primarily on the derivation and computation of the leading order corrections (order $N^{-2}$ ), due to microstructure, of both the real and imaginary parts of the effective indexes of the leaky modes of such averaged profiles. Since the leading order behavior is given by a homogenized (in angle) effective medium, we refer to this expansion as a homogenization expansion. Results on first-order corrections to homogenized eigenvalues of periodic composite media were obtained in $[13,10]$.

A few of the consequences of the homogenization expansion and numerical implementation that we wish to highlight are:

(1) Computational algorithm: Our analytic theory leads to a natural efficient computational algorithm for the modes and spectral characteristics, e.g. leakage rates, dispersion. Multiscale analysis enables us to eliminate the "stiff" aspects of the computation due to the rapidly varying structure.

(2) Arbitrary geometry and index contrasts: Although we require $N$-fold symmetry of the structure, the individual microfeatures may have arbitrary geometry and large index contrasts. In our current implementation, we approximate an arbitrary microfeature by a simple layered structure, having the local character of the structure of figure 1.

(3) Very good agreement with full numerical simulation: As illustrated in section 3 numerical simulations based on our theory give very good agreement with full numerical simulations [11], [16] of the complex effective indexes and, in particular, their imaginary parts, which correspond to the leakage rates. One expects homogenization theory to be valid when the wavelength of light is long compared with the individual microfeatures. We find very good agreement of our theory with full simulations even in regimes where $\lambda_{\mathrm{fs}} / d$, the ratio of wavelength to length scale of microfeatures, is as small as $3 / 2$. Although, the expansion is derived for $N$ large, with the $N=\infty$ limit being the leading order term, examples of section 3 show agreement in cases where $N=3$ and 
$N=6$. We also observe the expected departure of our approximate methods from the results of full simulations for sufficiently small $\lambda_{\mathrm{fs}} / d$.

(4) Sensitivity of leakage rates to microstructure: In section 3 we have used our theory to compute the first two nontrivial terms of the effective indexes (scattering resonances) of the leaky modes. The first term corresponds to an average theory and the second term is a correction due to microstructure. The imaginary parts of the effective indexes, corresponding to the leakage rates, are very sensitive to the introduction of microstructure and their accurate approximation requires both terms. In contrast, as noted above, the real parts of the effective indexes are relatively insensitive to microstructure, and are well captured by the leading order term.

(5) Corrected fields: We find that corrections, due to microstructure, of the mode fields predicted by the averaged structure are compactly supported in space if the microstructure perturbation is compactly supported in space. This is not possible for the true solution of the mathematical model. A consequence of this is that in the regime where certain interference effects are important, the expansion of this paper may have limited use. The rigorous theory of [5], which reproduces the second order homogenization expansion of modes and effective indices for $N$ large, does not have this limitation.

Mathematically, the leaky modes and effective indexes we compute are solutions to the scalar approximation to Maxwell's equations, in which the transverse components $\varphi$ of the electric field satisfy

$$
\left(\Delta_{\perp}+k^{2} n^{2}\right) \varphi=\beta^{2} \varphi
$$

where $\Delta_{\perp}$ denotes the Laplace operator in the transverse variables, $x_{\perp}=$ $\left(x_{1}, x_{2}\right)$. We introduce the notation $V=k^{2}\left(n_{g}^{2}-n^{2}\right)$ and $E=k^{2} n_{g}^{2}-\beta^{2}$, where $n_{g}$ denotes a background refractive index. For example, in the case of a glass waveguide with airholes, the refractive index, $n$, takes on the value $n_{g} \sim 1.45$ in glass and $n_{h} \sim 1$ in air. Thus, $V$ is a compactly supported potential for which

$$
V(\mathbf{x}) \geq 0 \text {, for all } \mathbf{x} \text { and } V(\mathbf{x})=0 \text {, for } r \geq r_{*} \text {. }
$$

The equation for $\varphi$ and $\beta$ can be viewed as a Schrödinger equation with potential $V\left(\mathbf{x}_{\perp}\right)$ and energy parameter $E$ :

$$
\mathcal{L} \varphi \equiv\left(-\Delta_{\perp}+V\right) \varphi=E \varphi \text {. }
$$

The potential, $V$, therefore does not support bound states (guided modes) and only has scattering states (radiation modes), along with scattering resonances (leaky modes).

Since we are interested in the escape of energy from the "core" we require that 
$\varphi$ satisfy an outgoing radiation condition as $r \rightarrow \infty$. This scattering resonance eigenvalue problem is non-selfadjoint and therefore can be expected to have complex eigenvalues, $E$, which determine the complex effective indexes $n_{\text {eff }} \equiv$ $\beta / k$. The imaginary parts of $n_{\text {eff }}$ govern the leakage rates for the waveguide [14].

\section{Multiple scales and homogenization expansion}

We now present a multiple scale expansion of solutions to the resonance problem for microstructures. A homogenized (averaged) theory occurs at leading order followed by systematically computable corrections. We carry out the analysis for a class of potentials $V$ with the dependence: $V=V(r, \theta, N \theta)=$ $V(r, \theta, \Theta)$, where $V$ is $2 \pi$-periodic in $\theta$ and $\Theta$. This allows both a slow and a fast angular modulation of the index profile. In the special case where $V$ does not depend on $\theta$ and we have $V=V(r, N \theta)$, the potential corresponds to an index profile with an $N$ - fold symmetry. Assume $V \geq 0$ and that for $r \geq r_{L}$, $V \equiv 0$.

Homogenization Expansion: The scattering resonance problem has, for large $N$, solutions with the formal expansion $\varphi(r, \theta ; N)=\Phi^{(N)}(r, \theta, \Theta)$, with $\Theta=N \theta$, given by

$$
\Phi^{(N)}=\Phi_{0}+\frac{1}{N^{2}} \Phi_{2}+\mathcal{O}\left(\frac{1}{N^{3}}\right) ; \quad E^{(N)}=E_{0}+\frac{1}{N^{2}} E_{2}+\mathcal{O}\left(\frac{1}{N^{3}}\right) .
$$

- $\left(\Phi_{0}(r, \theta), E_{0}\right)$ is a non-trivial solution of the resonance problem $\mathcal{L}_{\mathrm{av}} \Phi_{0}=$ $E_{0} \Phi_{0}$, where $\Phi_{0}$ is subject to the outward going radiation condition, and $\mathcal{L}_{\mathrm{av}}$ is determined by averaging $\mathcal{L}$ over $\Theta$.

- $\Phi_{2}=\Phi_{2}^{(p)}+\Phi_{2}^{(h)}$, where $\Phi_{2}^{(p)}$ is the mean zero solution (easily derived from the Fourier series of $V(\cdot, \cdot, \Theta)$ ) of

$$
\frac{1}{r^{2}} \partial_{\Theta}^{2} \Phi_{2}^{(p)}=\left[V(r, \theta, \Theta)-V_{\mathrm{av}}(r, \theta)\right] \Phi_{0}(r, \theta)
$$

and $\left(\Phi_{2}^{(h)}, E_{2}\right)$ solves:

$$
\begin{aligned}
& \left(\mathcal{L}_{\mathrm{av}}-E_{0}\right) \Phi_{2}^{(h)}= \\
& \quad\left(E_{2}+\frac{r^{2}}{2 \pi} \int_{0}^{2 \pi}\left|\partial_{\Theta}^{-1}\left[V(r, \theta, \Theta)-V_{\mathrm{av}}(r, \theta)\right]\right|^{2} d \Theta\right) \Phi_{0}(r, \theta)
\end{aligned}
$$

Finally, $E_{2}$ is uniquely determined by the condition that (2.3) has a solution which satisfies an outgoing radiation condition at $r=\infty$. 


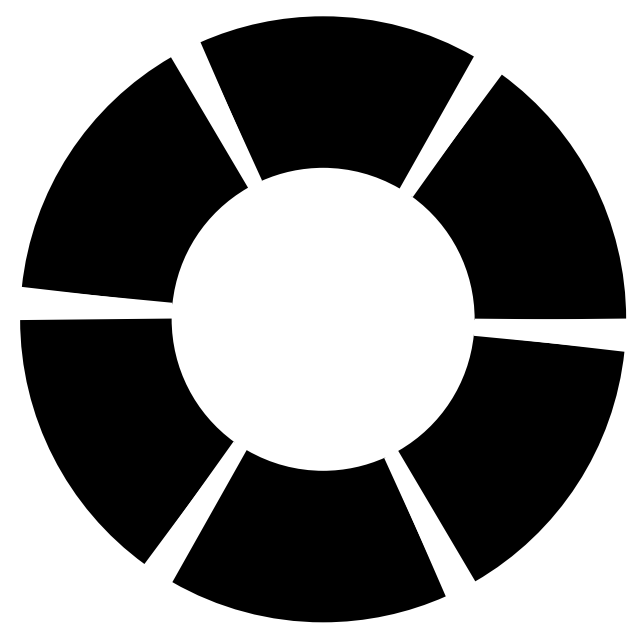

(a)

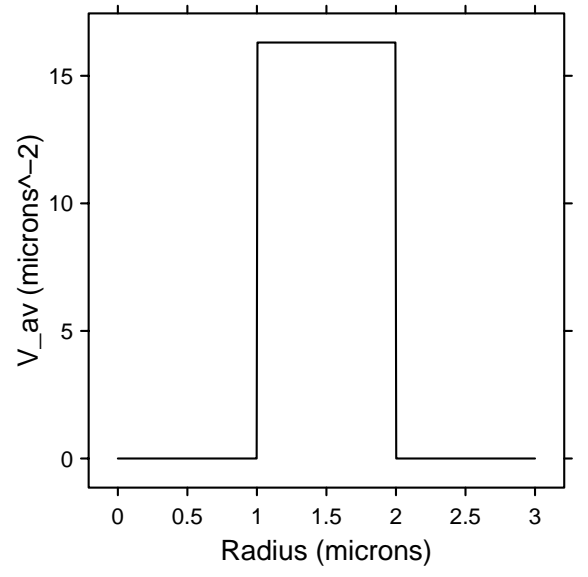

(b)

Fig. 1. (a) A ring of "air wedges" supported by $N=6$ webs of glass. The inner and outer radii of the annulus are 1 and $2 \mu \mathrm{m}$, respectively, while the air fill fraction within the annulus is $f=0.9$. (b) The averaged potential $V_{\mathrm{av}}$.

- This yields an approximate solution of the scalar wave equation and an approximation (neglecting vector effects) of the transverse electric field, $\overrightarrow{\mathcal{E}}=$ $\left(\mathcal{E}_{\perp}, \mathcal{E}_{\text {lon }}\right)=\left(\mathcal{E}_{1}, \mathcal{E}_{2}, \mathcal{E}_{3}\right)$, of Maxwell's equations:

$\mathcal{E}_{\perp, q}(\mathbf{x} ; \beta) \sim e^{i\left(\beta x_{3}-\omega t\right)}\left[\Phi_{0}\left(\left|\mathbf{x}_{\perp}\right|, \theta ; \omega\right)+\frac{1}{N^{2}} \Phi_{2}\left(\left|\mathbf{x}_{\perp}\right|, \theta, N \theta ; \omega\right)+\mathcal{O}\left(\frac{1}{N^{3}}\right)\right]$

Here, $\omega=c k$ and $\beta=\sqrt{k^{2} n_{g}^{2}-E}=\Re \beta+i \Im \beta$, with $\Im \beta>0 . \mathcal{E}_{\perp, q}\left(\mathbf{x}_{\perp}, x_{3}\right)$ decays with increasing $x_{3}$ and is therefore a "leaky mode." These modes are not square integrable.

\section{Numerical simulations for selected structures}

We now illustrate the theory of the preceding section with numerical calculations performed for two classes of structures: an annulus of air supported with glass "webs" (figure 1) and a subset of a hexagonal lattice (figure 2). We approximated the second structure by a simple layered potential (the websupported annulus is a simple layered structure without approximation). We took the refractive index of glass to be 1.45 and assumed the holes were empty.

We computed resonances of a structure of the type shown in figure 1 with $R_{\text {in }}=1 \mu \mathrm{m}, R_{\text {out }}=2 \mu \mathrm{m}$ for the fill fractions $f=0.8,0.9$, and 1 , with $N=3$ and $N=6$ holes. The calculations were performed for a range of free-space wavelengths $\lambda_{\mathrm{fs}}$ ranging from $1 \mu \mathrm{m}$ to $2 \mu \mathrm{m}$. The structure with $f=1$ is an idealized ring of air with no supporting structure. In figure 3 we compare the results of our theory to parallel calculations performed in [11] by a Fourier 


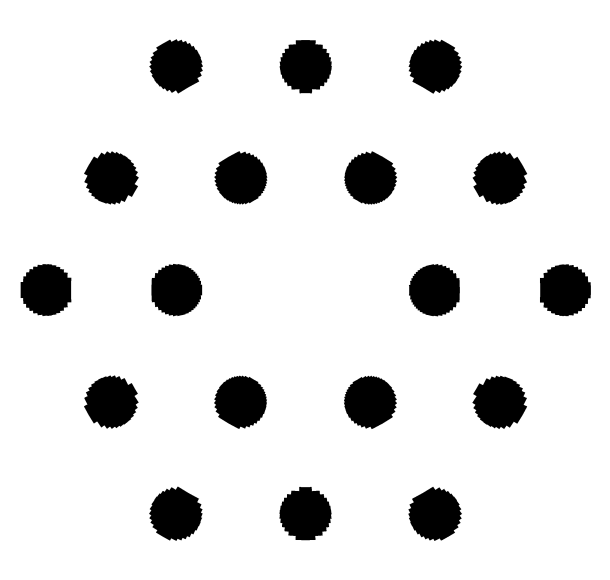

(a)

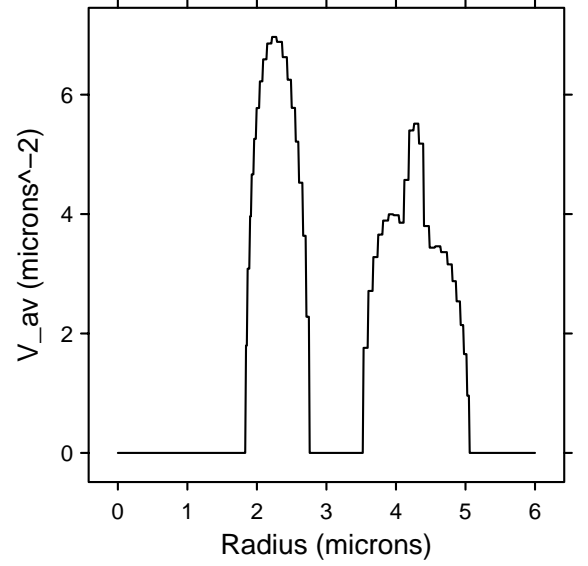

(b)

Fig. 2. (a) An 18-hole subset of a hexagonal lattice $(N=6)$ with inter-hole spacing $\Lambda=2.3 \mu \mathrm{m}$ and hole radius $R_{\text {hole }}=0.46 \mu \mathrm{m}$, approximated by a simple layered structure with $L=51$ layers. (b) The averaged potential $V_{\mathrm{av}}$ in units of $(\mu m)^{-2}$ vs. radius in units of $\mu m$.

decomposition algorithm. We observe that the two methods agree exactly for the $f=1$ case, as they should. Additionally, in all cases our method agrees with the Fourier calculation as $\lambda_{\mathrm{fs}} \rightarrow 2 \mu \mathrm{m}$. Even for smaller $\lambda_{\mathrm{fs}}$ the agreement is quite good except for the $f=0.8, N=3$ case, which is not surprising given the small value of $N$ and the fact that the width of the ring is equal to the smallest free space wavelength considered. The plots displayed in figure 3 are consistent with the expectation that approximation by the homogenization expansion improves (a) for fixed $\lambda_{\mathrm{fs}}$ and increasing $N$, as well as (b) for fixed $N$ and $\lambda_{\text {fs }}$ increasing.

The dashed lines in figure 3 display the attenuations of the averaged structures, without the $\mathcal{O}\left(N^{-2}\right)$ correction. The necessity of including these corrections is evident, though the effect in these structures is not as dramatic as in some of the other structures we consider, including that of figure 2 .

Another result reported in [11] is the effective index of the first excited state above the fundamental resonance (the $\mathrm{LP}_{11}$ state) for the $f=0.9, N=3$ structure at $\lambda_{\mathrm{fs}}=1.55 \mu \mathrm{m}$, which they found to be $1.255+0.00075 i$, which gives rise to a loss of $27 \mathrm{~dB} / \mathrm{mm}$. Our method predicts an effective index of $1.252+0.00083$, or a loss of $29 \mathrm{~dB} / \mathrm{mm}$. By comparison, this mode of the averaged structure has an effective index of $1.247+0.00044 i$, or an attenuation of $15 \mathrm{~dB} / \mathrm{mm}$. This illustrates the sensitivity of loss rates to the introduction of microstructure and the need for the first correction $N^{-2} E_{2}$ of our expansion.

Finally, we consider the structure depicted in figure 2. This is an 18-hole subset of a hexagonal lattice $(N=6)$ with inter-hole spacing $\Lambda=2.3 \mu \mathrm{m}$ and hole radius $R_{\text {hole }}=.46 \mu \mathrm{m}$. We found that the fundamental $\left(\mathrm{LP}_{01}\right)$ resonance has a 
leakage rate of $14 \mathrm{~dB} / \mathrm{cm}$, while that of the averaged structure was $0.92 \mathrm{~dB} / \mathrm{cm}$. By comparison, [16] found this rate to be $16 \mathrm{~dB} / \mathrm{cm}$ by solving the full vector problem with outgoing radiation conditions using a multipole method. In this example, the effect of the $N^{-2} E_{2}$ microstructure correction on the leakage rates is even more apparent than in the structure of figure 1. However, we cannot say how much of the discrepancy between [16] and ours is due to vector effects, and how much to the other approximations in our method. The role of vector effects is currently under investigation.

\section{Discussion}

The homogenization expansion can be viewed in the more general context of perturbation theory of "scattering resonances" for wave equations with rapidly varying perturbations which are not necessarily pointwise small. In quantum mechanics, the eigenvalue problem (1.3) is referred to as the scattering resonance problem. The complex eigenvalues are called scattering resonances or scattering frequencies, whose imaginary parts give the lifetime of elementary particle states. In a forthcoming paper [5] we develop a rigorous perturbation theory of such scattering resonances, valid for high contrast potentials (arbitrary index contrasts), which gives insight into the regime of validity of the homogenization expansion and provides concrete error bounds for the truncated expansion. The theory explains the trends observed in the simulations we report in section 3; for example, that our homogenization expansion provides an increasingly accurate approximation of the modes and effective indexes: (a) for fixed wavelength, $\lambda_{\mathrm{fs}}$, and $N$ increasing, and (b) for fixed $N$ and $\lambda_{\mathrm{fs}}$ increasing. The goal of this analytical work is to develop an understanding of the interplay among wavelength, spatial variation of the index contrast, and the geometry of the microstructure in determining the propagation properties

of microstructure waveguides and, in particular, in determining the regime of validity of the homogenization expansion.

\section{Acknowledgments}

The authors wish to thank B. Eggleton, R. Windeler, R. Bise, D. DiGiovanni and J. Jaspara for introducing them to the subject of microstructured optical fiber and for discussions on physical aspects. The authors also wish to thank R.V. Kohn, G.C. Papanicolaou, T. Salamon and M. Vogelius for stimulating and informative discussions during the preparation of this work. 


\section{References}

[1] A. Argyros, I.M. Bassett, M.A. van Eijkelenborg, M.C.J. Large, J. Zagari, N.A.P. Nicorovici, R.C. McPhedran, and C.M. de Sterke, Ring structures in microstructured polymer optical fibers, Optics Express 9 (2001), 813-820.

[2] A. Bensoussan, J.L. Lions, and G.C. Papanicolaou, Asymptotic analysis for periodic structures, Studies in Mathematics and its Applications, vol. 5, NorthHolland, 1978.

[3] R.F. Cregan, B.J. Managan, J.C. Knight, T.A. Birks, P.St.J. Russell, P.J. Roberts, and D.C. Allen, Single mode photonic band gap guidance of light in air, Science 285 (1999), 1537-1539.

[4] B.J. Eggleton, P.S. Westbrook, C.A. White, C. Kerbage, R.S. Windeler, , and G.L. Burdge, Cladding-mode-resonances in air-silica microstructure optical fibers, J. Lightwave Technol. 18 (2000), 1084-1100.

[5] S.E. Golowich and M.I. Weinstein, Scattering resonances and homogenization theory, Bell Labs preprint, 2002.

[6] S.E. Golowich and M.I. Weinstein, Homogenization expansion for resonances of microstructured photonic waveguides, J. Opt. Soc. Am. B (2003, to appear).

[7] J. Jasapara, R. Bise, and R. Windeler, Chromatic dispersion measurements in a photonic bandgap fiber, OFC, 2002.

[8] G.W. Milton, The theory of composites, Cambridge Monographs in Applied and Computational Mathematics, Cambridge, 2002.

[9] T.M. Monro, D.J. Richardson, N.G.R. Broderick, and P.J. Bennett, Modeling large air fraction holey optical fibers, J. Lightwave Tech. 18 (2000), 50-56.

[10] S. Moskow and M. Vogelius, First-order corrections to the homogenized eigenvalues of a periodic composite medium. a convergence proof, Proc. Roy. Soc. Edinburgh 127A (1997), 1263-1299.

[11] L. Poladian, N.A. Issa, , and T.M. Monro, Fourier decomposition algorithm for leaky modes of fibres with arbitrary geometry, Optics Express 10 (2002), 449-454.

[12] J.K. Ranka, R.S. Windeler, and A.J. Stentz, Visible continuum generation in air-silica microstructure optical fibers with anomolous dipersion at $800 \mathrm{~nm}$, Opt. Lett. 25 (2000), 25-27.

[13] F. Santosa and M. Vogelius, First-order corrections to the homogenized eigenvalues of a periodic composite medium, SIAM J. Appl. Math. 53 (1993), $1636-1668$.

[14] A. W. Snyder and John D. Love, Optical waveguide theory, Chapman and Hall, London, 1983. 
[15] M.J. Steel and R.M. Osgood, Elliptical hole photonic crystal fibers, Optics Letters 26 (2001), 229-231.

[16] T.P. White, R.C. McPhedran, C.M. de Sterke, L.C. Botten, and M.J. Steel, Confinement losses in microstructured optical fibers, Opt. lett. 26 (2001), 16601662. 


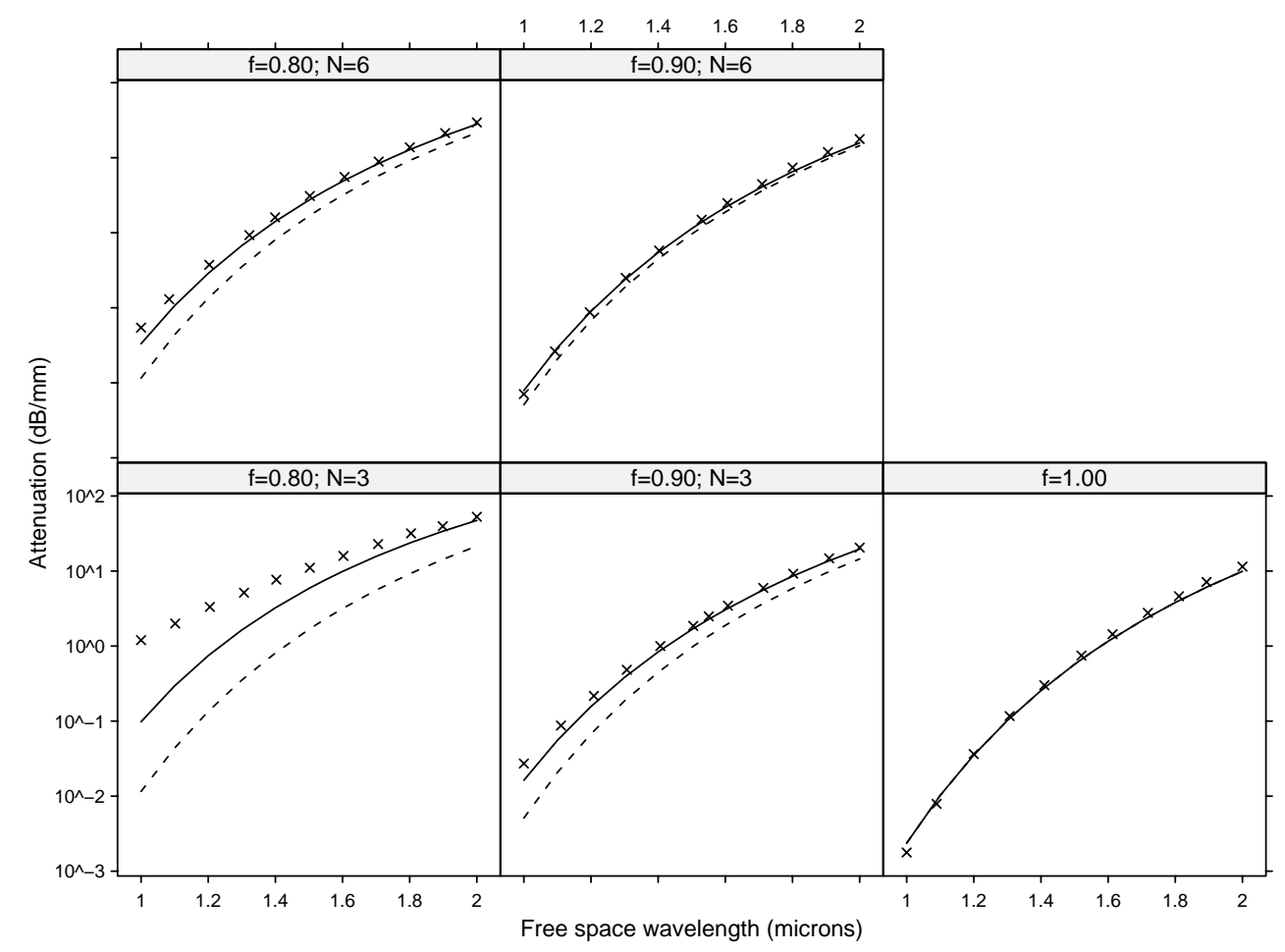

Fig. 3. Attenuation of the lowest order (fundamental, or $\mathrm{LP}_{01}$ ) resonance for a structure of the type shown in figure 1 , with $R_{\text {in }}=1 \mu \mathrm{m}, R_{\text {out }}=2 \mu \mathrm{m}$ for the fill fractions $f=0.8,0.9$, and 1 , with $N=3$ and $N=6$ holes. The calculations were performed for a range of free-space wavelengths $\lambda_{\mathrm{fs}}$ ranging from $1 \mu \mathrm{m}$ to $2 \mu \mathrm{m}$. The solid lines are the attenuations computed according to the methods of this paper, including the $\mathcal{O}\left(N^{-2}\right)$ corrections; the dashed lines are the attenuations of the averaged structure; and the crosses correspond to the results presented in figure $3 \mathrm{a}$ of [11]. 